\title{
New insights into PDAC growth promotion via a BAG3-mediated paracrine loop
}

$\mathrm{N}$ ew research published in Nature Communications has shown that Bcl-2-associated athanogene 3 (also known as BAG family molecular chaperone regulator 3 or BAG3) is an important factor in the development and growth of pancreatic ductal adenocarcinoma (PDAC) via the activation of stromal macrophages. The new findings highlight the potential of BAG3 as a novel therapeutic target.

Pancreatic cancer is one of the most lethal malignancies, and PDAC is the most common type of pancreatic cancer (accounting for the majority of exocrine tumours). Given the poor prognosis of patients with PDAC, improved understanding of tumour biology is urgently needed to identify novel therapeutic targets.

\section{4 ...antibody-treated mice showed a marked reduction in the number of stromal macrophages... 77}

\footnotetext{
"BAG3 was first described as a co-chaperone of HSP70, and the interaction between the two proteins is known to be crucial in mechanisms that sustain cell survival in cancer cells," explain authors Maria Caterina Turco and Vincenzo De Laurenzi. "Indeed, BAG3 is constitutively expressed in several primary tumours or tumour cell lines, where it has been shown to exert a prosurvival role through mechanisms that vary according to the cellular context," they add. Previous work had shown that BAG3 expression levels in PDAC correlated with patient survival, and that serum levels of BAG3 had potential as a serum biomarker. In this new work, Turco, De Laurenzi and colleagues wanted to explore how BAG3 was involved in tumour development.
}

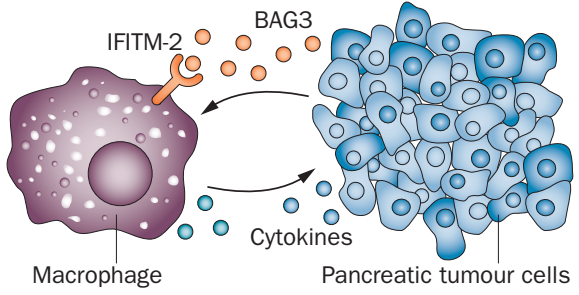

BAG3 activates stromal macrophages, thus promoting PDAC growth. Image produced in consultation with M. De Marco and M. C. Turco.

First, the investigators examined extracellular release of BAG3 from five different pancreatic cancer cell lines. All tested cells expressed intracellular BAG3, and the protein was released into the cell culture supernatant and was present in exosomes. This released BAG3 did not act as an autocrine factor; no binding of BAG3 to PDAC cell surfaces could be detected. However, in vitro studies revealed that BAG3 bound to the surface of mouse macrophages and primary human monocytes, activating these cells.

Further experiments demonstrated that BAG3-activated macrophages then released factors that promote PDAC cell proliferation in vitro, such as IL-6, which is known to have a role in tumour development. Using a variety of techniques, including western blotting, liquid chromatography-tandem mass spectometry and co-immunoprecipitation assays, the cell surface receptor for BAG3 was identified-interferon-induced transmembrane protein 2 (IFITM-2). Moreover, PI3K and p38 MAPK signalling pathways were found to be activated upon BAG3-IFITM-2 binding.

Importantly, an anti-BAG3 antibody blocked BAG3 activity on macrophages in vitro and reduced tumour growth as well as prevented metastasis in mouse models in vivo. "This antibody blocked tumour growth in [three] different animal models, including a model of a patient-derived xenograft and a syngeneic model, this last model is of great importance since the mice have an intact immune system," notes Turco. Depending on the mouse model used, tumour volumes were reduced by up to $70 \%$ and no peritoneal metastases were recorded in the antibody-treated mice.

Finally, in line with their hypothesized mechanism of action of BAG3 via stromal macrophage activation, analysis of tumour biopsy samples from control (phosphate buffered saline only) mice compared with antibody-treated mice showed a marked reduction in the number of stromal macrophages and a general decrease in macrophage-released cytokines in the latter group. Furthermore, BAG3 expression correlated with the number of infiltrating macrophages in tumour samples from nine patients with stage 3 PDAC.

"We showed that pancreatic adenocarcinoma cells secrete BAG3 that acts as a cytokine activating a paracrine loop," says Turco. "This biological activity of the BAG3 protein was completely unknown ... and these findings identify a novel mechanism that promotes tumour growth and the metastatic process."

The authors plan further work to study the various signalling pathways activated by BAG3 in macrophages, potentially expanding their research into other immune cells and the tumour microenvironment. The researchers are also in the early stages of developing a humanized version of the [anti-BAG3] antibody with the potential to be used in clinical trials as, according to the new study findings, BAG3 seems to be a promising target for PDAC therapy.

Katrina Ray

Original article Rosati, A. et al. BAG3 promotes pancreatic ductal adenocarcinoma growth by activating stromal macrophages. Nat. Commun. 6, 8695 (2015)

Further reading Erkan, M. et al. The role of stroma in pancreatic cancer: diagnostic and therapeutic implications. Nat. Rev. Gastroenterol. Hepatol. 9, 454-467 (2012) 